\title{
Intravenous patient-controlled analgesia does not increase the risk of postoperative delirium compared to patient-controlled epidural analgesia: a propensity score-matched retrospective cohort study
}

\author{
Hirotsugu Miyoshi ${ }^{1 \#}$, Ryuji Nakamura ${ }^{1 \#}$, Yuko Noda ${ }^{1}$, Hiroshi Yokomi ${ }^{1}$, Satoshi Kamiya ${ }^{1}$, Atsushi Morio ${ }^{1}$, \\ Tomoyuki Watanabe ${ }^{1}$, Soushi Narasaki ${ }^{1}$, Yukari Toyota ${ }^{1}$, Noboru Saeki ${ }^{1}$, Hiroshi Hamada ${ }^{2}$, \\ Yasuo M. Tsutsumi ${ }^{1}$ \\ ${ }^{1}$ Department of Anesthesiology and Critical Care, Hiroshima University, Hiroshima, Japan; ${ }^{2}$ Department of Anesthesiology and Palliative Medicine, \\ Tokyo Medical University, Tokyo, Japan \\ Contributions: (I) Conception and design: H Miyoshi, R Nakamura, T Watanabe, S Narasaki, Y Toyota, N Saeki; (II) Administrative support: H \\ Hamada, YM Tsutsumi; (III) Provision of study materials or patients: Y Noda, H Yokomi, S Kamiya, A Morio; (IV) Collection and assembly of data: \\ H Miyoshi, Y Noda, H Yokomi, S Kamiya, A Morio, T Watanabe; (V) Data analysis and interpretation: H Miyoshi, R Nakamura; (VI) Manuscript \\ writing: All authors; (VII) Final approval of manuscript: All authors. \\ \#These authors contributed equally to this work. \\ Correspondence to: Hirotsugu Miyoshi. Department of Anesthesiology and Critical Care, Hiroshima University, 1-2-3 Kasumi, Minami-ku, Hiroshima \\ 734-8551, Japan. Email: h-miyoshi@hiroshima-u.ac.jp.
}

Backgroundk It is unclear whether the doses of opioids and the routes of administration used for postoperative analgesic management are associated with delirium. We aimed to compare the incidence of postoperative delirium (POD) between intravenous patient-controlled analgesia (IVPCA) and patientcontrolled epidural analgesia (PCEA) in patients who underwent postoperative analgesic management using opioids.

Methods: We retrospectively investigated surgical patients $(n=3,324)$ who received patient-controlled analgesia (PCA). Morphine was used for IVPCA, and fentanyl and ropivacaine were used for PCEA. The patients' background characteristics, perioperative management, presence of POD, and postoperative analgesia technique after IVPCA $(n=1,184)$ or PCEA $(n=2,140)$ were assessed. We divided the patients into IVPCA and PCEA groups and compared the incidence of POD by propensity score matching. We used the independent $t$-test for comparisons between the groups, and $\mathrm{P}<0.05$ as considered as statistically significant.

Results: POD was noted in a total of 125 patients (3.8\%); 55 patients (4.6\%) with IVPCA and 70 patients $(3.3 \%)$ with PCEA $(\mathrm{P}=0.046)$. There was no statistically significant difference in cumulative opioid usage up to postoperative day 2 (in $\mathrm{mg}$ ) between patients with and without POD (POD 62.7 $\pm 39.8 v s$. non-POD $48.9 \pm 50.3, \mathrm{P}=0.10)$. After propensity score matching, 1,156 patients with similar baseline characteristics were selected. POD was noted in 22 of 578 patients (3.8\%) in the IVPCA group and 30 of 578 patients $(5.2 \%)$ in the PCEA group, with no difference between the two groups $(\mathrm{P}=0.256)$. On the other hand, opioid usage was higher in the IVPCA group than in the PCEA group $(\mathrm{P}<0.001)$.

Conclusions: There was no difference in the incidence of POD between morphine IVPCA and fentanyl PCEA when the patient characteristics were matched using propensity score matching. POD occurs regardless of the route and dose of opioid administration.

Keywords: Patient-controlled analgesia (PCA); postoperative delirium (POD); epidural analgesia; opioid

Submitted Apr 30, 2021. Accepted for publication Aug 19, 2021.

doi: 10.21037/apm-21-1084

View this article at: https://dx.doi.org/10.21037/apm-21-1084 


\section{Introduction}

Delirium is a major concern during the perioperative period and has been reported to increase mortality and hospital stay (1). Various factors such as old age and preoperative cognitive decline have been reported to affect delirium during hospitalization $(2,3)$. In non-cardiac surgery, postoperative delirium (POD) occurs in $5-15 \%$ of patients $(4,5)$. Since POD can be suppressed by early intervention, it is important to predict patients at high risk of POD before surgery $(6,7)$. With regard to risk factors for POD, opioid use and pain have been reported to cause POD $(8,9)$. Therefore, a balance between the two is important for postoperative management $(10,11)$. If severe pain is anticipated after surgery, such as thoracic or abdominal surgery, sufficient analgesia is required (12). With regard to postoperative analgesia, thoracic or abdominal surgery is a good indication for patient-controlled epidural analgesia (PCEA) (13). In addition, it is known that the addition of opioid to epidural anesthesia enhances the analgesic effect (14), and therefore, opioid is used for PCEA when an enhanced postoperative analgesic effect is required. As an analgesic method other than PCEA, intravenous patientcontrolled analgesia (IVPCA) is performed by intravenous administration of opioids.

Although various studies have reported the superiority of each analgesic method (10,15-17), the differences in the effects of each postoperative analgesic method on the development of POD are not yet fully understood. In some reports, the use of opioids is believed to be a risk factor for delirium $(18,19)$. Therefore, IVPCA with high opioid use is expected to have a higher risk of POD than PCEA. On the other hand, epidural analgesia increases the analgesic effect by using an opioid in combination with local anesthesia and reduces systemically administered opioids, but the addition of opioids to epidural anesthesia may also increase POD (20). However, it is unclear how the dose and route of administration of these opioids actually affects the development of POD.

To clarify this question, we investigated the effect of the route of administration, that is, IVPCA or PCEA, and the dose of opioid on the incidence of POD in patients undergoing postoperative analgesia with patient-controlled analgesia (PCA) using opioids. In addition, patient characteristics are strongly associated with POD. Previous studies reporting the effects of postoperative analgesia on POD might not have eliminated the impact of patient characteristics due to the small sample size of these studies.
To solve these problems, we compared IVPCA and PCEA in a relatively large population with controlled background factors. In our study, confounding factors that could affect the results of the multivariate analysis were efficiently removed by propensity score matching (21). We present the following article in accordance with the STROBE reporting checklist (available at https://dx.doi.org/10.21037/apm-21-1084).

\section{Methods}

\section{Study design and etbical considerations}

This was a propensity score-matched retrospective cohort study. The study was conducted in accordance with the Declaration of Helsinki (as revised in 2013). The study was approved by the institutional ethics board of Hiroshima University Hospital (No. E-1462) and individual consent for this retrospective analysis was waived.

\section{Patients selection criteria}

This study is a single-center study conducted at Hiroshima University Hospital. After obtaining IRB approval, we retrospectively investigated the medical records of 3,601 patients (IVPCA: 1,383, PCEA: 2,218) who underwent various surgeries and received PCA between January 1, 2009 and December 31, 2013. We excluded patients with inflammatory bowel disease, those who underwent liver transplantation, and those who continued with tracheal intubation after surgery. In addition, we excluded patients who were unable to continue PCEA due to epidural catheter problems (spontaneous removal, inappropriate range of analgesic effect, etc.) from the study. In other words, we excluded 101 inflammatory bowel disease, 80 liver transplant surgery, 10 continuation of tracheal intubation, and 71 patients who had trouble with the epidural catheter, and finally examined 3,324 patients (IVPCA: 1,184, PCEA: 2,140). In addition, we matched the patient backgrounds of IVPCA and PCEA by propensity score matching for 3,324 patients, and 1,156 patients (IVPCA: 578, PCEA: 578) were included in the post-match subjects. A flowchart of the patient selection process is illustrated in Figure 1.

\section{Post-operative pain management}

In our hospital, PCEA was the first choice for postoperative analgesia, and IVPCA was chosen when PCEA was not 


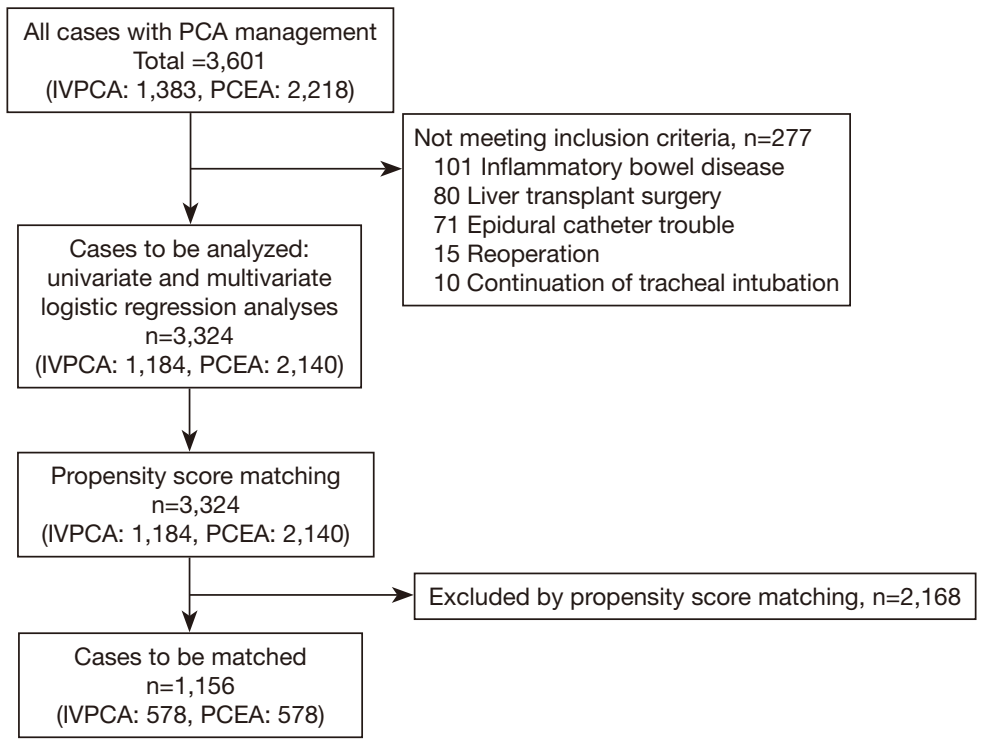

Figure 1 Patient selection criteria. PCA, patient-controlled analgesia; IVPCA, intravenous patient-controlled analgesia; PCEA, patientcontrolled epidural analgesia.

possible due to the use of oral anticoagulants or patient request. Morphine was routinely used for IVPCA, while fentanyl and ropivacaine were used for PCEA. We used CADD-Legacy ${ }^{\circledR}$ PCA Model 6300 (Smiths Medical, St Paul, MN, USA) or i-Fusor ${ }^{\mathrm{TM}}$ Puls (JMS, Hiroshima, Japan) for the PCA infusion pump. PCA chemical solutions and pump settings were as follows: IVPCA: morphine $(1 \mathrm{mg} / \mathrm{mL})$, no background infusion, a demand dose of $1 \mathrm{mg} / \mathrm{push}$, and a lockout interval of $5 \mathrm{~min}$; PCEA: $0.15 \%$ ropivacaine with $2 \mu \mathrm{g} / \mathrm{mL}$ fentanyl (for patients $<80$ years old) or $1 \mu \mathrm{g} / \mathrm{mL}$ (for patients $>80$ years old), background infusion rate of $3 \mathrm{~mL} / \mathrm{h}$, demand dose of $2 \mathrm{~mL} /$ push, and lockout interval of $15 \mathrm{~min}$.

As a standard PCEA management protocol, the continuous dose was reduced to $1 \mathrm{~mL} / \mathrm{h}$ on the $3 \mathrm{rd}$ postoperative day, and the catheter was removed in the morning of the 4th postoperative day. We set the observation period up to 4 days after surgery as the observation period for postoperative analgesia. Movement pain visual analogue scale (VAS) scores while walking for 0 to $100 \mathrm{~mm}$ and rest VAS scores were measured twice daily from day 1 to day 4 after the surgery.

\section{Definition and diagnosis of delirium}

Delirium was defined as clouding of consciousness (visual and auditory hallucinations), restlessness, decreased activity, or dangerous behavior consistent with the Diagnostic and
Statistical Manual of Mental Disorders (DSM-IV) criteria while using PCA. Delirium was assessed twice daily by our acute pain service team along with pain assessment. Postoperative analgesia continued for at least 4 days postoperatively, regardless of IVPCA or PCEA; therefore, our study evaluated delirium during this period.

\section{Surveyed factors}

We investigated the method of PCA, VAS score for postoperative pain from 0 to $100 \mathrm{~mm}$, the presence or absence of POD, and the postoperative day of onset of POD. Pain VAS scores were investigated at rest and during movement. To show the cumulative opioid doses of IVPCA and PCEA, we calculated morphine $15 \mathrm{mg}$ and fentanyl $0.3 \mathrm{mg}$ as an equipotent value, and converted PCEA fentanyl usage into morphine and tabulated it (22).

We also investigated patient background factors, including age, sex, height, body weight, body mass index (BMI), coexisting diseases such as dementia, cerebrovascular disorder, respiratory disorders such as asthma or chronic obstructive pulmonary disease (COPD), diabetes mellitus, hypertension, liver dysfunction, renal dysfunction, anemia, smoking history, and surgical factors including operation time (minutes) and amount of blood loss $(\mathrm{mL})$. We also investigated the surgical site and divided the patients into four groups: upper abdomen, lower abdomen, thoracic, and 
Table 1 The postoperative day of delirium onset and the number of occurrences of delirium (\%) after the application of IVPCA or PCEA

\begin{tabular}{lcccc}
\hline Postoperative day & IVPCA $(n=1,184),(\%)$ & PCEA $(n=2,140)(\%)$ & All patients $(n=3,324)(\%)$ & P value \\
\hline 0 & $6(0.5)$ & $18(0.8)$ & $24(0.7)$ & 0.82 \\
1 & $13(1.1)$ & $23(1.1)$ & $36(1.1)$ & 0.01 \\
2 & $25(2.1)$ & $20(0.9)$ & $45(1.4)$ & $<0.01$ \\
3 & $7(0.6)$ & $6(0.3)$ & $13(0.4)$ & 0.01 \\
4 & $4(0.3)$ & $3(0.1)$ & $7(0.2)$ & $<0.01$ \\
Total & $55(4.6)$ & $70(3.3)$ & $125(3.8)$ & $<0.01$ \\
\hline
\end{tabular}

The postoperative day of onset of delirium and the number of occurrences of delirium (\%) are shown. The incidence of postoperative delirium was high on the 1st and 2nd postoperative days and low on the 3rd and 4th postoperative days. Statistical analysis was performed for all patients using chi-square test and residual analysis and a $\mathrm{P}$ value $<0.05$ was considered to indicate statistical significance. IVPCA, intravenous patient-controlled analgesia; PCEA, patient-controlled epidural analgesia.

others, depending on the surgical site.

\section{Statistical analysis}

In the first step, we conducted a comprehensive survey of factors associated with the development of POD in all study patients. We adopted the VAS and opioid values on the second day after surgery. We divided patients into two groups, with and without POD, and performed univariate and multivariate logistic regression analyses. In the multivariable logistic regression analysis, the candidates for dependent factors were all the factors investigated in this study. First, in order to eliminate multicollinearity, only one of the factors with a strong correlation was introduced into the model. The variables were stepwise eliminated when Wald statistic was 1.0 or less. Finally, it was confirmed that the number of factors that remained was $1 / 10$ or less of the number of POD occurrences. We calculated the odds ratio (OR) of every 10 years for age and of every hour for operation time.

In the second step, we divided the patients based on the PCA route, to investigate the effect of postoperative analgesic technique on the incidence of POD. First, we divided the patients into the IVPCA and PCEA groups, and calculated the propensity score using logistic regression. In calculating the propensity score, the candidates for dependent factors were all the factors measured in the preoperative and intraoperative periods. After elimination of multicollinearity, the variables were eliminated stepwisely by Wald statistic. Finally, it was confirmed that the number of factors remaining was $1 / 10$ or less of the number of IVPCA or PCEA cases. Then, 1:1 matching was performed using the nearest neighbor matching method with a propensity score. After 1:1 propensity score matching, we compared both groups. Patient characteristic factors other than opioid doses in both groups were matched. That is, the statistical differences between the two groups for patients' background including age, sex, height, body weight, coexisting diseases such as dementia, cerebrovascular disorder, respiratory disorders such as asthma or COPD, diabetes mellitus, hypertension, liver dysfunction, renal dysfunction, anemia, smoking, and surgical factors including operation time and blood loss amount were corrected. On the other hand, opioid doses were not corrected because IVPCA patients had higher opioid doses than PCEA patients.

Regarding the analysis, the independent $t$-test was used to compare continuous variables, and the chi-squared test was used to compare categorical variables between the two groups. A P value $<0.05$ was considered to indicate statistical significance.

\section{Results}

A total of 3,601 patients were included at baseline. Thereafter, 277 patients were excluded from the analysis. Finally, a total of 3,324 patients (IVPCA: 1,184, PCEA: $2,140)$ were investigated. POD was noted in 125 patients (3.8\%); 55 (4.6\%) with IVPCA and 70 (3.3\%) with PCEA $(\mathrm{P}=0.046)$. The postoperative day on which delirium developed is shown in Table 1.

Tables 2 and 3 show the results of a comprehensive survey of factors associated with the development of POD in all patients. Table 2 shows the results of a univariate analysis of various factors in both patient groups based on the presence 
Table 2 Univariate analysis of patient characteristics according to the presence and absence of postoperative delirium

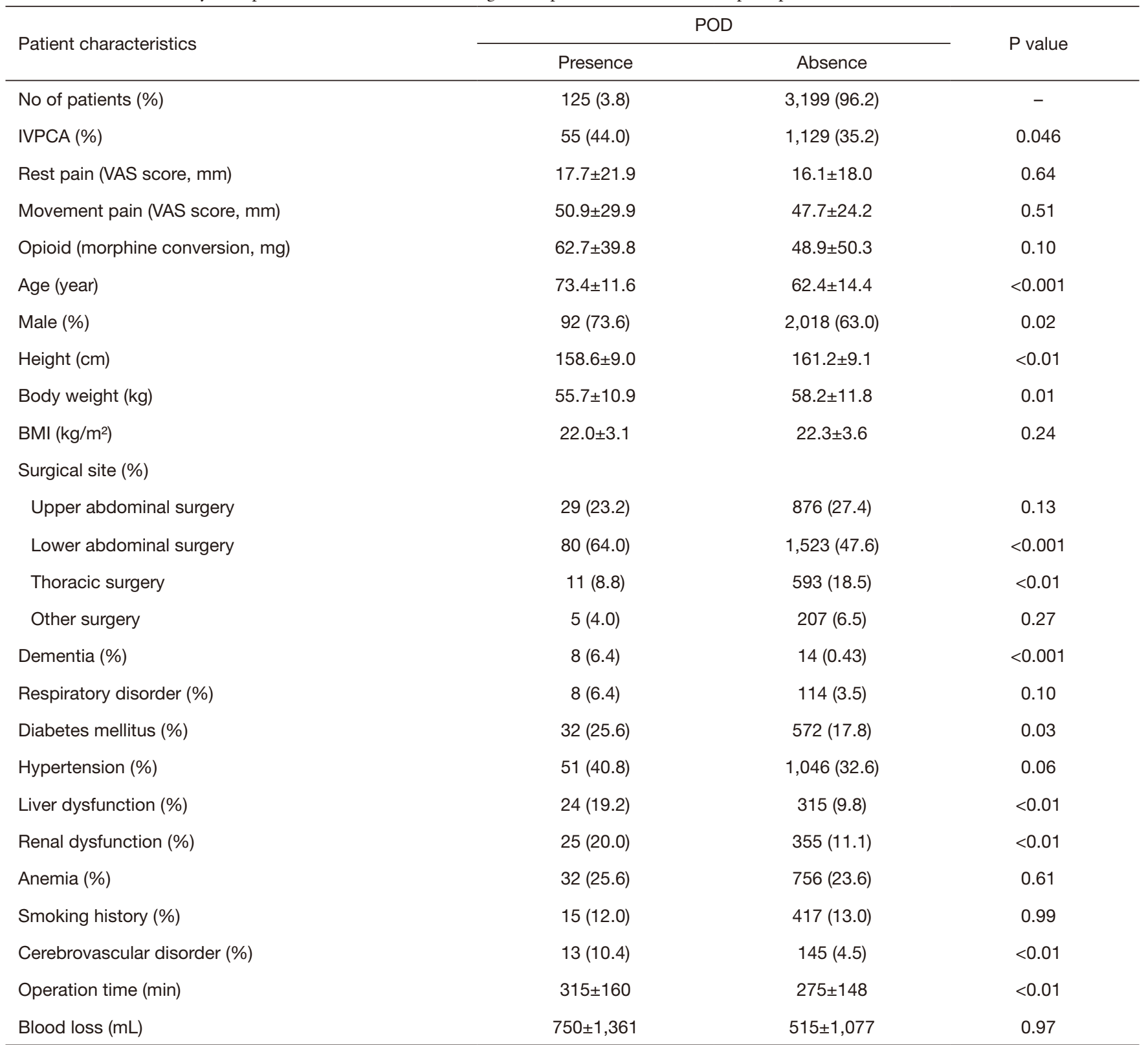

A comparison of patient characteristics according to the presence and absence of postoperative delirium is shown. A $P$ value $<0.05$ was considered to indicate statistical significance. IVPCA, intravenous patient-controlled analgesia; VAS, visual analogue scale; BMI, body mass index; POD, postoperative delirium.

or absence of POD. Although there were some statistically significant differences in some background factors, there was no statistical difference in pain and opioid use between the two groups. Table 3 shows the results of the logistic regression analysis with crude data. In this analysis, the risk factors found to be significantly associated with POD were IVPCA, age, sex, dementia, liver dysfunction, and operation time.
In a comprehensive survey, there was a significant difference in the development of POD depending on the route of PCA, although it was weak. Therefore, propensity score matching was performed to confirm whether the route of PCA was associated with the development of POD. Table 4 shows the patient characteristics before and after propensity score matching between the IVPCA and 
Table 3 Multiple logistic regression analysis of variables responsible for postoperative delirium

\begin{tabular}{lcc}
\hline Patient characteristics & OR $(95 \% \mathrm{Cl})$ & P value \\
\hline IVPCA & $1.53(1.05-2.24)$ & 0.03 \\
Age (/10 years) & $2.41(1.95-2.99)$ & $<0.001$ \\
Gender (if male) & $1.58(1.03-2.42)$ & 0.04 \\
Body weight (/kg) & $0.96(0.91-1.02)$ & 0.20 \\
Dementia & $8.50(3.25-22.2)$ & $<0.001$ \\
Liver dysfunction & $2.19(1.34-3.5 .7)$ & $<0.01$ \\
Cerebrovascular disorder & $1.50(0.80-2.81)$ & 0.21 \\
Operation time (/hour) & $1.14(1.06-1.23)$ & $<0.001$ \\
\hline
\end{tabular}

In the multivariable logistic regression, the dependent variables examined were IVPCA, patient background factors, and surgical factors, while the objective variable was postoperative delirium. A P value $<0.05$ was considered to indicate statistical significance. OR, odds ratio; $\mathrm{Cl}$, confidence interval; IVPCA, intravenous patient-controlled analgesia.

PCEA groups. After matching, there was no difference in patient characteristics between the two groups, and POD was noted in 52 of $1,156(4.5 \%)$ matched patients, of which $22 / 578(3.8 \%)$ in the IVPCA group and $30 / 578(5.2 \%)$ in the PCEA group had POD.

\section{Discussion}

We investigated the incidence of POD in 3,324 postoperative patients who underwent pain management with opioid PCA, and POD was noted in a total of 125 patients (3.8\%); 55 patients $(4.6 \%)$ with IVPCA and 70 patients $(3.3 \%)$ with PCEA. In this study, $80 \%$ of patients with POD developed it within two days after surgery, consistent with the report by Marcantonio et al. (23). In this study, we used propensity score matching to match patient backgrounds in a relatively large population of patients who received IVPCA or PCEA in order to investigate whether morphine IVPCA or fentanyl PCEA was superior in causing POD. In the univariate analysis, $\mathrm{POD}$ was more common in patients who underwent IVPCA. However, after propensity score matching, there was no difference in the incidence of delirium in the patients receiving IVPCA and PCEA. Although not statistically significant, the incidence of POD was higher in PCEA than in IVPCA. These results suggest that morphine IVPCA itself does not increase the incidence of POD compared to fentanyl PCEA.
There are many factors that cause delirium, and it is unknown whether opioids themselves cause delirium. In some reports, opioids have been reported as risk factors for delirium (24). Various studies have been conducted on the administration route and dose of opioids in POD. If opioid doses are associated with delirium, patients with IVPCA are presumed to be at a higher risk of POD because it uses more opioids than PCEA. In abdominal surgery, which is the subject of our study, strong analgesia is required. In a study comparing the incidence of delirium after abdominal surgery, Mann et al. reported that the incidence of POD was not different between morphine IVPCA and sufentanil combined with bupivacaine PCEA in 70 patients (15). Eriksson-Mjöberg et al. compared morphine IVPCA and morphine PCEA, and reported that there was no difference in the incidence of POD between them (25). In addition to abdominal surgery, Inan et al. reported that morphine IVPCA had no difference in postoperative mental dysfunction compared to morphine PCEA (24). Previous studies have not shown that IVPCA increases the risk of delirium more than PCEA, despite lower doses of opioid being administered by PCEA. In our study, we compared the incidence of POD in morphine IVPCA and fentanyl PCEA, and showed that the amount of opioid use compared to morphine was lower in PCEA, but there was no difference in the incidence of POD between them. Furthermore, there was no significant difference in the amount of opioid used between the groups according to the presence or absence of POD. These facts suggest that there is no difference in the incidence of POD between opioid-based PCEA and IVPCA, and that PCEA can reduce opioid use but not the incidence of POD compared to IVPCA.

In this study, we assumed that fentanyl PCEA had a lower incidence of POD because the morphine-equivalent dose of fentanyl administered to the epidural space was less than that of morphine administered for IVPCA. However, there was no difference in the incidence of POD between the two groups in this study. Two types of opioids, morphine and fentanyl, were used in our research. It is known that there is a difference in the ability to induce POD depending on the type of opioid used for postoperative analgesia (16). Indeed, among opioids in postoperative analgesia, meperidine has been reported to increase the incidence of POD compared to other opioids such as morphine, regardless of the administration method $(11,26)$. However, morphine and fentanyl IVPCA have been reported to have no difference in POD incidence (27). We believe that the difference between 
Table 4 Comparison of patient characteristics of the IVPCA and PCEA groups before and after propensity score matching

\begin{tabular}{|c|c|c|c|c|c|c|}
\hline Patient characteristics & \multicolumn{3}{|c|}{ Before propensity score matching } & \multicolumn{3}{|c|}{ After propensity score matching } \\
\hline No of patients & 2,140 & 1,184 & & 578 & 578 & \\
\hline POD (\%) & $70(3.2)$ & $55(4.6)$ & 0.046 & $30(5.2)$ & $22(3.8)$ & 0.256 \\
\hline Rest pain (VAS score, mm) & $17.0 \pm 18.8$ & $17.5 \pm 19.1$ & 0.080 & $25.8 \pm 18.1$ & $24.7 \pm 19.0$ & 0.463 \\
\hline Opioid (morphine conversion, mg) & $21.8 \pm 9.9$ & $72 \pm 65.5$ & $<0.001$ & $41.9 \pm 31.3$ & $15.9 \pm 2.9$ & $<0.001$ \\
\hline Age (year) & $64.4 \pm 13.0$ & $59.9 \pm 16.3$ & $<0.01$ & $62.9 \pm 14.0$ & $62.8 \pm 15.0$ & 0.858 \\
\hline Gender (No. of male, \%) & 1,332 (62.2) & $778(65.7)$ & 0.047 & $380(65.7)$ & $363(62.8)$ & 0.297 \\
\hline Height (cm) & $160.7 \pm 9.0$ & $161.7 \pm 9.2$ & $<0.01$ & $161.0 \pm 9.1$ & $161.2 \pm 9.1$ & 0.739 \\
\hline \multicolumn{7}{|l|}{ Surgical site (\%) } \\
\hline Upper abdominal surgery & $498(23.0)$ & $474(40.0)$ & $<0.01$ & $133(23.0)$ & $242(41.9)$ & $<0.01$ \\
\hline Lower abdominal surgery & $1,031(48.2)$ & $505(42.7)$ & & $259(44.8)$ & $223(38.6)$ & \\
\hline Thoracic surgery & $493(23.0)$ & $111(9.4)$ & & $146(25.3)$ & $63(10.9)$ & \\
\hline Other surgery & $118(5.5)$ & $94(7.9)$ & & $40(7.0)$ & $50(8.7)$ & \\
\hline Dementia (\%) & $11(0.5)$ & $11(0.9)$ & 0.158 & $5(0.8)$ & $3(0.5)$ & 0.478 \\
\hline Respiratory disorder (\%) & $94(4.4)$ & $28(2.4)$ & $<0.01$ & $21(3.6)$ & $17(2.9)$ & 0.509 \\
\hline Diabetes mellitus (\%) & $353(16.5)$ & $251(21.2)$ & $<0.01$ & $111(19.2)$ & $116(20)$ & 0.711 \\
\hline Cerebrovascular disorder (\%) & $72(3.4)$ & $86(7.3)$ & $<0.01$ & $33(5.7)$ & $30(5.2)$ & 0.697 \\
\hline Operation time (min) & $271.3 \pm 129.8$ & $287.1 \pm 178.2$ & $<0.01$ & $272.1 \pm 139.4$ & $272.6 \pm 148.7$ & 0.953 \\
\hline Blood loss (mL) & $398.8 \pm 642.8$ & $750.2 \pm 1584.9$ & $<0.01$ & $461.0 \pm 782.3$ & $459.6 \pm 619.3$ & 0.974 \\
\hline
\end{tabular}

A comparison of the patient characteristics of the IVPCA and PCEA groups before and after propensity score matching is shown. We did not match opioid doses in the IVPCA and PCEA groups because in almost all cases, opioid doses were higher in IVPCA patients than in PCEA patients. A $P$ value $<0.05$ was considered to indicate statistical significance. POD, postoperative delirium; IVPCA, intravenous patient-controlled analgesia; PCEA, patient-controlled epidural analgesia; VAS, visual analogue scale; BMI, body mass index.

morphine and fentanyl is unlikely to affect the incidence of POD. One of the reasons why fentanyl PCEA did not suppress the development of POD despite the low-dose opioids could be the effect of the chemical properties of fentanyl administered into the epidural space. Since fentanyl is a highly lipophilic opioid, it is easily translocated into the intravascular compartment when administered into the epidural space (28). In particular, in the case of continuous administration, it was reported that the blood fentanyl concentrations were similar regardless of the intravenous or epidural administration (29-31). In this study, it is possible that PCEA fentanyl was absorbed into the blood and acted 
on the central nervous system to cause delirium.

Since POD is significantly associated with age-related changes such as preoperative cognitive decline and cerebrovascular disease (17), it is very important to adjust patient background factors when conducting comparative studies. In particular, since POD is more likely to occur in elderly patients, it may be necessary to compare the frequency of POD with respect to the diversity of the elderly patients' background. Our study showed that IVPCA had a higher POD frequency when comparing the POD frequency of IVPCA and PCEA without controlling the patients' background. However, on controlling patients' background by propensity score matching, there was no difference in POD frequency between the two groups. These results suggest that patients who received IVPCA developed POD more frequently because they were affected by the patient background factors. In other words, IVPCA itself does not increase the risk of POD compared to PCEA when patient background factors are comparable.

Regarding the limitations of this study, we investigated major risk factors for POD, but not all perioperative factors. For example, age and preoperative cognitive impairment have been reported to be major factors associated with POD, and our results were strongly consistent with these points (2,32). However, the type of anesthetic used during surgery and the depth of anesthesia have also been reported to affect POD, but these factors were not considered in this study (33). The relationship between POD and other perioperative factors that we have not investigated should be evaluated in future studies. Regarding the effect of surgical site on POD, logistic regression analysis showed that surgical site was not a risk factor for developing POD. Therefore, we did not include surgical site as a factor to be adjusted by propensity score matching. However, in the results after matching, the distribution of surgical sites was different between the two groups, and in particular, lower abdominal surgery was more common in the PCEA group. In univariate analysis, lower abdominal surgery had a higher incidence of POD than other surgical sites, which may have increased the incidence of POD in the PCEA group.

\section{Conclusions}

There was no difference in the incidence of POD between morphine IVPCA and fentanyl PCEA when the patient characteristics were matched by propensity score matching. POD occurs regardless of the route and dose of opioid administration.

\section{Acknowledgments}

The authors acknowledge the support of the postoperative pain management team of Hiroshima University Hospital. Funding: None.

\section{Footnote}

Reporting Checklist: The authors have completed the STROBE reporting checklist. Available at https://dx.doi. org/10.21037/apm-21-1084

Data Sharing Statement: Available at https://dx.doi. org/10.21037/apm-21-1084

Peer Review File: Available at https://dx.doi.org/10.21037/ apm-21-1084

Conflicts of Interest: All authors have completed the ICMJE uniform disclosure form (available at https://dx.doi. org/10.21037/apm-21-1084). The authors have no conflicts of interest to declare.

Ethical Statement: The authors are accountable for all aspects of the work in ensuring that questions related to the accuracy or integrity of any part of the work are appropriately investigated and resolved. The study was conducted in accordance with the Declaration of Helsinki (as revised in 2013). The study was approved by the institutional ethics board of Hiroshima University Hospital (No. E-1462) and individual consent for this retrospective analysis was waived.

Open Access Statement: This is an Open Access article distributed in accordance with the Creative Commons Attribution-NonCommercial-NoDerivs 4.0 International License (CC BY-NC-ND 4.0), which permits the noncommercial replication and distribution of the article with the strict proviso that no changes or edits are made and the original work is properly cited (including links to both the formal publication through the relevant DOI and the license). See: https://creativecommons.org/licenses/by-nc-nd/4.0/.

\section{References}

1. Witlox J, Eurelings LS, de Jonghe JF, et al. Delirium in elderly patients and the risk of postdischarge mortality, institutionalization, and dementia: a meta-analysis. JAMA 
2010;304:443-51.

2. Ansaloni L, Catena F, Chattat R, et al. Risk factors and incidence of postoperative delirium in elderly patients after elective and emergency surgery. Br J Surg 2010;97:273-80.

3. Jones RN, Fong TG, Metzger E, et al. Aging, brain disease, and reserve: implications for delirium. Am J Geriatr Psychiatry 2010;18:117-27.

4. Marcantonio ER, Goldman L, Mangione CM, et al. A clinical prediction rule for delirium after elective noncardiac surgery. JAMA 1994;271:134-9.

5. Demeure MJ, Fain MJ. The elderly surgical patient and postoperative delirium. J Am Coll Surg 2006;203:752-7.

6. Inouye SK, Bogardus ST Jr, Charpentier PA, et al. A multicomponent intervention to prevent delirium in hospitalized older patients. N Engl J Med 1999;340:669-76.

7. Aldecoa C, Bettelli G, Bilotta F, et al. European Society of Anaesthesiology evidence-based and consensus-based guideline on postoperative delirium. Eur J Anaesthesiol 2017;34:192-214.

8. Leung JM, Sands LP, Paul S, et al. Does postoperative delirium limit the use of patient-controlled analgesia in older surgical patients? Anesthesiology 2009;111:625-31.

9. Vaurio LE, Sands LP, Wang Y, et al. Postoperative delirium: the importance of pain and pain management. Anesth Analg 2006;102:1267-73.

10. Fong HK, Sands LP, Leung JM. The role of postoperative analgesia in delirium and cognitive decline in elderly patients: a systematic review. Anesth Analg 2006;102:1255-66

11. Morrison RS, Magaziner J, Gilbert M, et al. Relationship between pain and opioid analgesics on the development of delirium following hip fracture. J Gerontol A Biol Sci Med Sci 2003;58:76-81.

12. Lynch EP, Lazor MA, Gellis JE, et al. The impact of postoperative pain on the development of postoperative delirium. Anesth Analg 1998;86:781-5.

13. Block BM, Liu SS, Rowlingson AJ, et al. Efficacy of postoperative epidural analgesia: a meta-analysis. JAMA 2003;290:2455-63.

14. Fischer RL, Lubenow TR, Liceaga A, et al. Comparison of continuous epidural infusion of fentanyl-bupivacaine and morphine-bupivacaine in management of postoperative pain. Anesth Analg 1988;67:559-63.

15. Mann C, Pouzeratte Y, Boccara G, et al. Comparison of intravenous or epidural patient-controlled analgesia in the elderly after major abdominal surgery. Anesthesiology 2000;92:433-41.

16. Swart LM, van der Zanden V, Spies PE, et al. The
Comparative Risk of Delirium with Different Opioids: A Systematic Review. Drugs Aging 2017;34:437-43.

17. Bilotta F, Lauretta MP, Borozdina A, et al. Postoperative delirium: risk factors, diagnosis and perioperative care. Minerva Anestesiol 2013;79:1066-76.

18. Moore AR, O'Keeffe ST. Drug-induced cognitive impairment in the elderly. Drugs Aging 1999;15:15-28.

19. Clegg A, Young JB. Which medications to avoid in people at risk of delirium: a systematic review. Age Ageing 2011;40:23-9.

20. Burton AW, Rajagopal A, Shah HN, et al. Epidural and intrathecal analgesia is effective in treating refractory cancer pain. Pain Med 2004;5:239-47.

21. D'Agostino RB Jr. Propensity scores in cardiovascular research. Circulation 2007;115:2340-3.

22. Treillet E, Laurent S, Hadjiat Y. Practical management of opioid rotation and equianalgesia. J Pain Res 2018;11:2587-601.

23. Marcantonio ER, Goldman L, Orav EJ, et al. The association of intraoperative factors with the development of postoperative delirium. Am J Med 1998;105:380-4.

24. Inan N, Cakan T, Ozen M, et al. The effect of opioid administration by different routes on the psychological functions of elderly patients. Agri 2007;19:32-8.

25. Eriksson-Mjöberg M, Svensson JO, Almkvist O, et al. Extradural morphine gives better pain relief than patientcontrolled i.v. morphine after hysterectomy. Br J Anaesth 1997;78:10-6.

26. Marcantonio ER, Juarez G, Goldman L, et al. The relationship of postoperative delirium with psychoactive medications. JAMA 1994;272:1518-22.

27. Herrick IA, Ganapathy S, Komar W, et al. Postoperative cognitive impairment in the elderly. Choice of patientcontrolled analgesia opioid. Anaesthesia 1996;51:356-60.

28. de Leon-Casasola OA, Lema MJ. Postoperative epidural opioid analgesia: what are the choices? Anesth Analg 1996;83:867-75.

29. Glass PS, Estok P, Ginsberg B, et al. Use of patientcontrolled analgesia to compare the efficacy of epidural to intravenous fentanyl administration. Anesth Analg 1992;74:345-51.

30. Loper KA, Ready LB, Downey M, et al. Epidural and intravenous fentanyl infusions are clinically equivalent after knee surgery. Anesth Analg 1990;70:72-5.

31. Sandler AN, Stringer D, Panos L, et al. A randomized, double-blind comparison of lumbar epidural and intravenous fentanyl infusions for postthoracotomy pain relief. Analgesic, pharmacokinetic, and respiratory effects. 
Anesthesiology 1992;77:626-34.

32. Koebrugge B, Koek HL, van Wensen RJ, et al. Delirium after abdominal surgery at a surgical ward with a high standard of delirium care: incidence, risk factors and outcomes. Dig Surg 2009;26:63-8.

Cite this article as: Miyoshi $\mathrm{H}$, Nakamura R, Noda Y, Yokomi H, Kamiya S, Morio A, Watanabe T, Narasaki S, Toyota Y, Saeki N, Hamada H, Tsutsumi YM. Intravenous patientcontrolled analgesia does not increase the risk of postoperative delirium compared to patient-controlled epidural analgesia: a propensity score-matched retrospective cohort study. Ann Palliat Med 2021;10(10):10160-10169. doi: 10.21037/apm-21-1084
33. Pandharipande PP, Pun BT, Herr DL, et al. Effect of sedation with dexmedetomidine vs lorazepam on acute brain dysfunction in mechanically ventilated patients: the MENDS randomized controlled trial. JAMA 2007;298:2644-53. 